Review Article

\title{
Pharmacological Actions, Molecular Mechanisms, Pharmacokinetic Progressions, and Clinical Applications of Hydroxysafflor Yellow A in Antidiabetic Research
}

\author{
Xilan Zhang $\mathbb{D}$, ${ }^{1}$ Dayue Shen $\mathbb{D}^{1},{ }^{1}$ Yating Feng $\mathbb{D},{ }^{1}$ Yuanping $\mathrm{Li} \mathbb{D},{ }^{2}$ and Hui Liao $\mathbb{D}^{2}$ \\ ${ }^{1}$ School of Pharmacy, Shanxi Medical University, Taiyuan 030001, China \\ ${ }^{2}$ Department of Pharmacy, Fifth Hospital of Shanxi Medical University (Shanxi Provincial People's Hospital), \\ Taiyuan 030012, China
}

Correspondence should be addressed to Hui Liao; huiliao@263.net

Received 29 July 2021; Revised 1 November 2021; Accepted 16 November 2021; Published 13 December 2021

Academic Editor: Kai Wang

Copyright (C) 2021 Xilan Zhang et al. This is an open access article distributed under the Creative Commons Attribution License, which permits unrestricted use, distribution, and reproduction in any medium, provided the original work is properly cited.

\begin{abstract}
Hydroxysafflor yellow A (HSYA), a nutraceutical compound derived from safflower (Carthamus tinctorius), has been shown as an effective therapeutic agent in cardiovascular diseases, cancer, and diabetes. Our previous study showed that the effect of HSYA on high-glucose-induced podocyte injury is related to its anti-inflammatory activities via macrophage polarization. Based on the information provided on PubMed, Scopus and Wanfang database, we currently aim to provide an updated overview of the role of HSYA in antidiabetic research from the following points: pharmacological actions, molecular mechanisms, pharmacokinetic progressions, and clinical applications. The pharmacokinetic research of HSYA has laid foundations for the clinical applications of HSYA injection in diabetic nephropathy, diabetic retinopathy, and diabetic neuropathy. The application of HSYA as an antidiabetic oral medicament has been investigated based on its recent oral delivery system research. In vivo and in vitro pharmacological research indicated that the antidiabetic activities of HSYA were based mainly on its antioxidant and anti-inflammatory mechanisms via JNK/c-jun pathway, NOX4 pathway, and macrophage differentiation. Further antiinflammatory exploration related to NF- $\kappa$ B signaling, MAPK pathway, and PI3K/Akt/mTOR pathway might deserve attention in the future. The anti-inflammatory activities of HSYA related to diabetes and diabetic complications will be a highlight in our following research.
\end{abstract}

\section{Introduction}

Hydroxysafflor yellow A (HSYA) is a single chalcone glycoside compound [1] which is derived from safflower (Carthamus tinctorius), a traditional Chinese herb (Figure 1) [2]. The most general and traditional method of extracting HSYA is water immersion. However, many other extraction systems have been developed such as smashing tissue extraction, microwave extraction, ultrasound extraction, and Soxhlet extraction [3]. HSYA has been commonly used in China to treat cardiovascular disease (CVD) [4]. Our recent literature research provides a number of articles and reviews describing novel applications of HSYA towards various diseases such as cancer and diabetes, beyond their conventional use against CVD (Figure 1).

According to the International Diabetes Federation, the number of adults diagnosed with diabetes has increased from 285 million in the year 2009 to 463 million in the year $2019,95 \%$ of which are type 2 diabetes mellitus (T2DM) patients [5]. Diabetes is associated with accelerated rates of macrovascular and microvascular complications [6]. Macrovascular complications affect the heart, brain, and peripheral arteries and are termed CVD, cerebrovascular disease, and peripheral vascular disease, respectively [7]. Diabetesrelated macrovascular complications are responsible for the impaired quality of life, disability, and premature death 


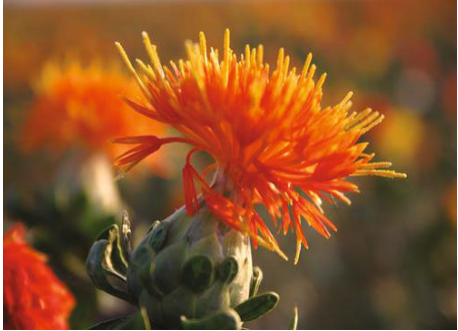

Carthamus tinctorius

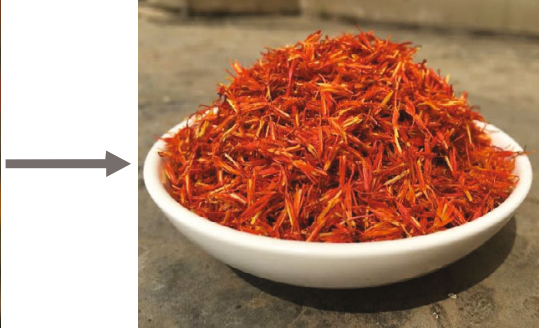

Carthami flos

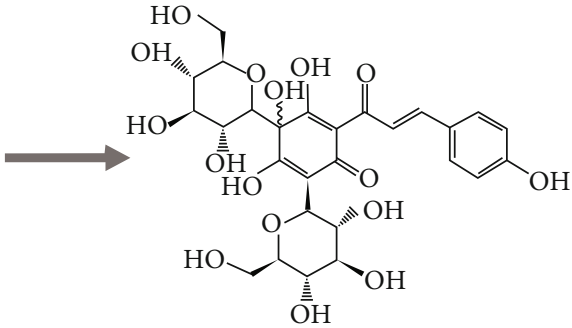

Hydroxysafflor yellow A

(a)

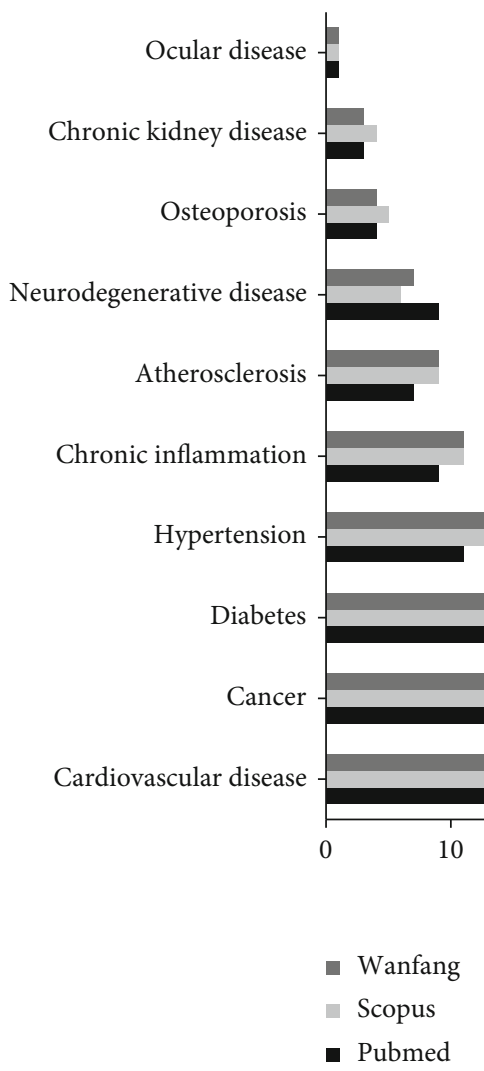

FIGURE 1: The source, structure, and literature research of hydroxysafflor yellow A: (a) the source and structure of hydroxysafflor yellow A; (b) the research articles of the top ten diseases related to hydroxysafflor yellow A. Abbreviation: Wanfang: Wanfang Data.

associated with diabetes $[8,9]$. Microvascular complications affect the retina which is the inner part of the eye, the kidneys, and peripheral nerves. The resulting conditions are known as diabetic retinopathy, diabetic nephropathy (DN), and diabetic neuropathy, respectively [7]. In a study involving 689 individuals with T2DM obtained at baseline, the occurrences of microvascular complications observed during a median follow-up of 10.5 years were as follows: 206 patients had DN, 161 patients had retinopathy, and 179 patients had neuropathy [10].

Specifically, T2DM is characterized by chronic systemic inflammation alongside hyperglycemia and insulin resistance in the body [11]. Clinical data analysis showed that elevated C-reactive protein (CRP), tumor necrosis factor(TNF-) $\alpha$, and interleukin- (IL-) 6 were the most common inflammation indicators in diabetes-related angiopathies
[12]. Related research suggested that HSYA could inhibit the apoptosis of pancreatic $\beta$-cells, and this might be the underlying mechanisms through which HSYA regulates glycolipid metabolism in T2DM rats [13]. Our previous study indicated that HSYA had direct protective effects on high glucose- (HG-) induced podocyte injury and indirect protective effects by regulating macrophage M1/M2 polarization [14]. These effects were related to its antioxidant and antiinflammatory activities in vitro [15].

In this article, we first reviewed the in vivo and in vitro antidiabetic pharmacological actions and antidiabetic molecular mechanisms of HSYA. Based on signal research in the application of HSYA in the treatment of inflammation-related diseases, possible anti-inflammatory pathways involved in antidiabetic effects were discussed. The clinical applications of HSYA in diabetic macrovascular 
and microvascular complications were then summarized based on its recent pharmacokinetic progression. Finally, possible application of HSYA as an antidiabetic oral medicament was investigated.

\section{Antidiabetic Pharmacological Research and Related Mechanisms}

2.1. In Vivo Antidiabetic Research. T2DM was induced in rats by feeding high-fat diet (HFD) for four weeks followed by intraperitoneal injection of streptozocin (STZ). The established models were treated with HSYA for eight weeks while metformin was used as positive control. The results showed that the underlying mechanisms of HSYA in T2DM rats were related to the following activities: the direct or indirect inhibition of pancreatic $\beta$-cell apoptosis, the improvement of insulin resistance, and the regulation of glycolipid metabolism [13].

Also, in the treatment of HFD- and STZ-induced T2DM rats by HSYA, renal protective effects were observed based on the improvement of renal functions including serum creatinine (Scr), blood urea nitrogen (BUN), glomerular volume, podocyte number, and cell apoptosis markers. Furthermore, in the HSYA treatment group, the levels of TNF- $\alpha$ and the inflammatory products, including free fatty acids (FFA) and lactic dehydrogenase (LDH), were significantly decreased. Regarding oxidative stress markers, the level of superoxide dismutase (SOD) markedly increased in the HSYA treatment group, while the level of malondialdehyde (MDA) in the serum and kidney tissue evidently decreased [16].

In STZ-induced type 1 diabetes mellitus (T1DM) rats, a diabetic wound model was established by full-thickness excisional wounds that extended through the panniculus carnosus with a biopsy punch. Topical application of HSYA significantly enhanced the wound closure rate, and the time taken for complete wound closure was 17 days, whereas 30 days was needed for complete wound closure with phosphate-buffered saline (PBS) treatment [17].

2.2. In Vitro Antidiabetic Research. In vitro antidiabetic studies were conducted on seven different cell lines: rat INS-1 insulinoma cells [18], mice MPC-5 podocyte cells [14], human umbilical vein endothelial cells (HUVECs) [17, 19], human brain microvascular endothelial cells (HBMECs) [20], 3T3-L1 preadipocytes and adipocytes [21], and RAW264.7 macrophage cells [14, 17].

The loss of functional insulin-producing $\beta$-cells is a hallmark of diabetes; therefore, understanding the cellular biology of the pancreas is crucial. Rat insulinoma INS- 1 cells are widely used to study glucose-stimulated insulin secretion [22]. DN is one of the microvascular complications of diabetes and is a main cause of end-stage nephropathy. The most common clinical feature of $\mathrm{DN}$ is progressive proteinuria which is related to podocyte function. Podocyte plays an important role in maintaining the integrity and function of the glomerular filtration barrier. MPC-5 cell line is also widely used to evaluate renal injury in vitro [23]. Related research showed that HG-induced apoptosis of podocytes and pancreatic $\beta$-cells was reversed by HSYA $[14,18]$.
In diabetic patients, hyperglycemia-induced endothelial injury results in all kinds of vascular complications [24]. In vitro research showed that HG increased HUVEC apoptosis, vascular permeability, monocyte adhesion, the formation of reactive oxygen species (ROS), and the expression of NADPH oxidase 4 (NOX4) protein. The increased vascular injury by HG was attenuated by HSYA [19]. Another in vitro research showed that HSYA could inhibit methylglyoxal-induced injury in cultured HBMEC, which was associated with its antiglycation effect. Methylglyoxal is mainly formed from the degradation of glucose and glycated proteins [20].

For both diabetic wounds and DN progression, a central feature is the persistence of chronic inflammation, which is partly due to the prolonged presence of proinflammatory macrophages [25, 26]. In HG- and lipopolysaccharide(LPS-) induced RAW264.7 macrophage cells, HSYA showed its anti-inflammatory effects by decreasing TNF- $\alpha$, IL- $1 \beta$, and nitric oxide (NO) levels $[14,17]$. From Table 1, we could see that the main antidiabetic mechanism of HSYA is through its anti-inflammatory activity.

2.3. Anti-Inflammatory Signals in Antidiabetic Research of HSYA. So far, our review has shown that the antidiabetic mechanisms of HSYA are related to the following signals: c-jun NH2-terminal kinases/c-jun (JNK/c-jun) pathway [18], NOX4 pathway [19], macrophage polarization [14], and phosphoinositide 3-kinase/protein kinase B (PI3K/ Akt) pathway [13]. HSYA also showed its ability to cause a decrease in oxidative stress factors such as ROS $[18,19]$ and hydrogen peroxide $\left(\mathrm{H}_{2} \mathrm{O}_{2}\right)$ [19].

Inflammation is closely linked to the pathogenesis of diabetes, and chronic inflammation is one of the main causes of insulin resistance. Proinflammatory mediators can be related to obesity and induce insulin resistance in adipose tissue. Signaling pathways of transcription factors, particularly nuclear factor- $\kappa \mathrm{B}(\mathrm{NF}-\kappa \mathrm{B})$ signaling, are involved in insulin sensitivity [27]. NF- $\kappa$ B plays a crucial role in the development of diabetic complications because of its involvement in the expression of genes that are responsible for the damage of organs such as the brain, liver, heart, muscles, endothelium, adipose tissue, and pancreas by inflammation, apoptosis, and oxidative stress [28].

The role of NF- $\kappa \mathrm{B}$ signal in the antidiabetic activities of HSYA has not been previously reported. Figure 2 indicates that NF- $\kappa \mathrm{B}$ signal plays an important role in the use of HSYA to treat other inflammatory diseases [29-38]. This might provide some research points to explore antiinflammatory mechanisms of HSYA in the treatment of diabetes and diabetes complications.

\subsection{Possible Anti-Inflammatory Mechanisms in Antidiabetic} Research of HSYA. In Figure 2, organ damages treated by HSYA via the NF- $\kappa$ B pathway include brain damages such as ischemia reperfusion-injury, traumatic brain injury, ischemic stroke, and Alzheimer's disease (AD) [29-32]; lung injury such as fetal lung fibroblasts, chronic obstructive pulmonary disease, and acute respiratory distress syndrome 
TABLE 1: Summary of pharmacological effects and mechanisms of HSYA on diabetes and diabetes complications.

\begin{tabular}{|c|c|c|c|c|c|c|c|c|}
\hline Disease & Species/strains & $\begin{array}{l}\text { Effective dose/ } \\
\text { concentration }\end{array}$ & Route & $\begin{array}{l}\text { Positive } \\
\text { control }\end{array}$ & $\begin{array}{l}\text { Intervention } \\
\text { time }\end{array}$ & $\begin{array}{l}\text { Main improved } \\
\text { results }\end{array}$ & $\begin{array}{c}\text { Mechanisms/ } \\
\text { pathways }\end{array}$ & Reference \\
\hline Diabetes & $\begin{array}{l}\text { HFD- and } \\
\text { STZ-induced } \\
\text { T2DM rats }\end{array}$ & $120 \mathrm{mg} / \mathrm{kg}$ & i.g. & $\begin{array}{l}\text { Metformin as } \\
\text { positive } \\
\text { control }\end{array}$ & 8 weeks & $\begin{array}{c}\text { Pancreatic } \beta \text {-cell } \\
\text { apoptosis } \downarrow \text {, FBG } \downarrow \text {, } \\
\text { IR } \downarrow \text {, TG } \downarrow \text {, TC } \downarrow \text {, } \\
\text { LDLC } \downarrow \text {, glycogen } \\
\text { synthase } \uparrow, \text { hepatic } \\
\text { glycogen } \uparrow\end{array}$ & $\begin{array}{l}\text { Regulation on } \\
\text { glycolipid } \\
\text { metabolism via } \\
\text { PI3K/Akt } \\
\text { pathway }\end{array}$ & [13] \\
\hline Diabetes & $\begin{array}{l}\text { HG-induced } \\
\text { rat INS-1 } \\
\text { insulinoma } \\
\text { cells } \\
\text { (pancreatic } \\
\beta \text {-cells) }\end{array}$ & $800 \mu \mathrm{M}$ & & $\begin{array}{c}\mathrm{N}- \\
\text { Acetylcysteine } \\
\text { as oxidative } \\
\text { stress } \\
\text { scavenger } \\
\text { control }\end{array}$ & 72 hours & $\begin{array}{c}\text { Pancreatic } \beta \text {-cell } \\
\text { apoptosis } \downarrow, \text { ROS } \downarrow \text {, } \\
\text { MDA } \downarrow, \text { CAT } \uparrow, \\
\text { GSH-px } \uparrow, \text { SOD } \uparrow\end{array}$ & $\begin{array}{l}\text { Antioxidative } \\
\text { effects via JNK/c- } \\
\text { jun pathway }\end{array}$ & [18] \\
\hline $\begin{array}{l}\text { Diabetic } \\
\text { nephropathy }\end{array}$ & $\begin{array}{l}\text { HFD- and } \\
\text { STZ-induced } \\
\text { T2DM rats }\end{array}$ & $120 \mathrm{mg} / \mathrm{kg}$ & i.g. & & 8 weeks & $\begin{array}{c}\mathrm{Scr} \downarrow, \mathrm{UN} \downarrow, \mathrm{TG} \downarrow \\
\mathrm{TC} \downarrow, \mathrm{LDLC} \downarrow, \mathrm{FBG} \downarrow \\
\mathrm{TNF}-\alpha \downarrow, \mathrm{LDH} \downarrow \\
\mathrm{FFA} \downarrow, \mathrm{MDA} \downarrow \\
\mathrm{SOD} \uparrow\end{array}$ & $\begin{array}{c}\text { Antioxidative and } \\
\text { anti- } \\
\text { inflammation } \\
\text { effects }\end{array}$ & [16] \\
\hline $\begin{array}{l}\text { Diabetic } \\
\text { nephropathy }\end{array}$ & $\begin{array}{l}\text { HG-induced } \\
\text { mice MPC-5 } \\
\text { podocyte cells } \\
\text { and HG- } \\
\text { induced mice } \\
\text { RAW264.7 } \\
\text { cells }\end{array}$ & $\begin{array}{l}100 \mu \mathrm{M} \\
200 \mu \mathrm{M}\end{array}$ & & $\begin{array}{l}\text { Kaempferol as } \\
\text { positive } \\
\text { control }\end{array}$ & 24 hours & $\begin{array}{c}\text { Podocyte apoptosis } \downarrow \\
\text { In podocytes: } \\
\text { TNF- } \alpha \downarrow \text {, IL- } 1 \beta \downarrow \\
\text { In RAW264.7 cells: } \\
\text { TNF- } \alpha \downarrow \text {, iNOS } \downarrow \text {, } \\
\text { IL- } 1 \beta \downarrow \text { CD } 206 \uparrow, \\
\text { Arg- } 1 \uparrow\end{array}$ & $\begin{array}{l}\text { Anti- } \\
\text { inflammation } \\
\text { effects directly on } \\
\text { podocyte cells } \\
\text { and indirectly via } \\
\text { macrophage } \\
\text { polarization }\end{array}$ & {$[14]$} \\
\hline $\begin{array}{l}\text { Diabetic } \\
\text { vascular } \\
\text { injury }\end{array}$ & $\begin{array}{l}\text { HG-induced } \\
\text { HUVECs }\end{array}$ & $\begin{array}{c}10 \mu \mathrm{M} \\
25 \mu \mathrm{M}, 50 \mu \mathrm{M}\end{array}$ & & & $\begin{array}{l}24,48 \text {, and } \\
72 \text { hours }\end{array}$ & $\begin{array}{c}\text { HUVEC } \\
\text { hyperpermeability } \downarrow \text {, } \\
\text { HUVEC apoptosis } \downarrow \text {, } \\
\text { VCAM-1 } \downarrow \text {, ICAM- } \\
\text { 1 } \downarrow \text {, E-selectin } \downarrow \text {, } \\
\text { NOX4 } \downarrow \text {, ROS } \downarrow \text {, } \\
\mathrm{H}_{2} \mathrm{O}_{2} \downarrow\end{array}$ & $\begin{array}{c}\text { Anti- } \\
\text { inflammation } \\
\text { effects via the } \\
\text { NOX4 pathway }\end{array}$ & [19] \\
\hline $\begin{array}{l}\text { Diabetic } \\
\text { vascular } \\
\text { injury }\end{array}$ & $\begin{array}{l}\text { Methylglyoxal- } \\
\text { induced } \\
\text { HBMECs }\end{array}$ & $\begin{array}{c}10,50, \text { and } \\
100 \mu \mathrm{M}\end{array}$ & & & 24 hours & $\begin{array}{c}\text { HBMEC apoptosis } \downarrow \text {, } \\
\text { caspase-3 } 3 \text {, AGEs } \downarrow\end{array}$ & $\begin{array}{l}\text { Antiglycation } \\
\text { effects }\end{array}$ & {$[20]$} \\
\hline $\begin{array}{l}\text { Diabetic } \\
\text { wound }\end{array}$ & $\begin{array}{l}\text { STZ-induced } \\
\text { T1DM rats }\end{array}$ & $2 \mathrm{mg} / \mathrm{mL}$ & vs ext & $\begin{array}{l}\text { Hydrogel as } \\
\text { positive } \\
\text { control }\end{array}$ & 30 days & $\begin{array}{c}\text { Wound closure } \uparrow \text {, } \\
\text { granulation tissue } \\
\text { formation } \uparrow \text {, collagen } \\
\text { disposition } \uparrow \text {, } \\
\text { secretion of VEGF } \uparrow \text {, } \\
\text { TGF- } \beta 1 \uparrow\end{array}$ & & [17] \\
\hline $\begin{array}{l}\text { Diabetic } \\
\text { wound }\end{array}$ & $\begin{array}{l}\text { HUVECs and } \\
\text { LPS-induced } \\
\text { RAW264.7 } \\
\text { cells }\end{array}$ & $\begin{array}{l}0.4,0.8, \text { and } \\
1.6 \mathrm{mM}\end{array}$ & & & $\begin{array}{l}60 \text { and } 96 \\
\text { hours }\end{array}$ & $\begin{array}{c}\text { NO production } \downarrow \text {, } \\
\text { HEK migration } \uparrow \text {, } \\
\text { HUVEC tube } \\
\text { formation } \uparrow\end{array}$ & $\begin{array}{c}\text { Anti- } \\
\text { inflammation } \\
\text { effects }\end{array}$ & [17] \\
\hline $\begin{array}{l}\text { Diabetic } \\
\text { obesity }\end{array}$ & $\begin{array}{c}\text { 3T3-L1 } \\
\text { preadipocytes } \\
\text { and adipocytes }\end{array}$ & $100 \mathrm{mg} / \mathrm{L}$ & & & 24 hours & $\begin{array}{c}\text { PPAR } \gamma 2 \text { promoter } \\
\text { activities } \uparrow, \\
\text { PPAR } \gamma 2 \uparrow\end{array}$ & $\begin{array}{l}\text { Increasing the } \\
\text { expression of } \\
\text { insulin signaling } \\
\text { pathway-related } \\
\text { genes }\end{array}$ & [21] \\
\hline
\end{tabular}

Abbreviations: T1DM: type 1 diabetes mellitus; T2DM: type 2 diabetes mellitus; HFD: high-fat diet; STZ: streptozotocin; FBG: fasting blood glucose; IR: insulin resistance; TG: triglyceride; TC: total cholesterol; LDLC: low-density lipoprotein cholesterol; DN: diabetic nephropathy; ROS: reactive oxygen species; SOD: superoxide dismutase; CAT: catalase; GSH-px: glutathione peroxidase; MDA: malondialdehyde; Scr: serum creatinine; UN: urea nitrogen; LDH: lactate dehydrogenase; FFA: free fatty acids; NOX4: NADPH oxidase 4; $\mathrm{H}_{2} \mathrm{O}_{2}$ : hydrogen peroxide; HG: high glucose; HBMECs: human brain microvascular endothelial cells; HUVECs: human umbilical vein endothelial cells; VCAM-1: vascular cell adhesion molecule-1; ICAM-1: intercellular adhesion molecule-1; iNOS: inducible nitric oxide synthase; TNF- $\alpha$ : tumor necrosis factor- $\alpha$; CD206: mannose receptor; Arg-1: arginase-1; IL-1 $\beta$ : interleukin- $\beta$; LPS: lipopolysaccharide; AGEs: advanced glycation end-products; VEGF: vascular growth factors; TGF- $\beta 1$ : transforming growth factor- $\beta 1$; NO: nitric oxide; HEKs: human epithelial keratinocytes; PPAR $\gamma 2$ : peroxisome proliferator-activated receptor- $\gamma 2$. 


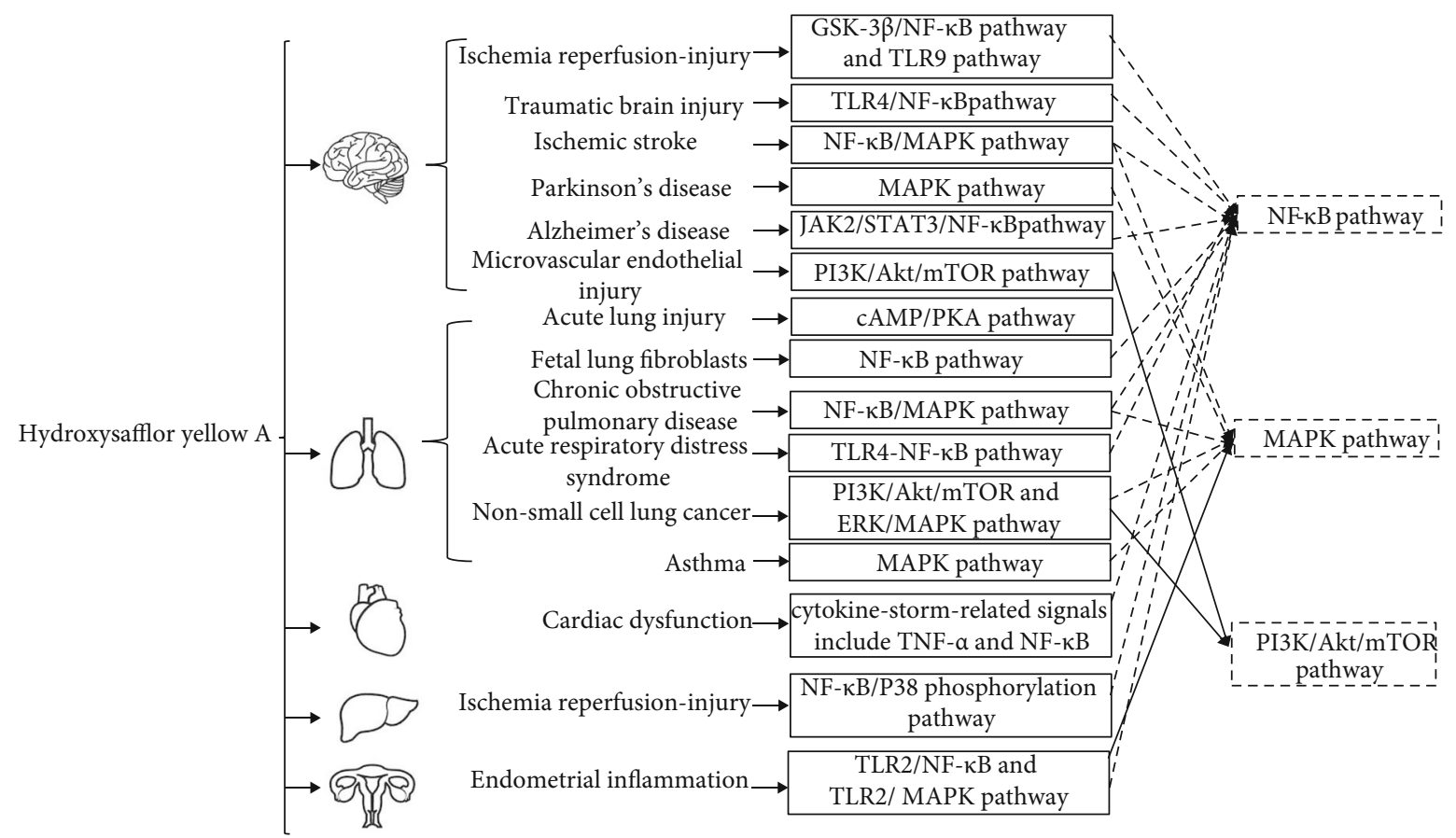

FIgURE 2: The molecular mechanisms of hydroxysafflor yellow A in the treatment of inflammation-related diseases. Abbreviations: GSK3 $\beta$ : glycogen synthase kinase-3 $\beta$; NF- $\kappa$ B: nuclear factor- $\kappa$ B; TLR9: toll-like receptor 9; TLR4: toll-like receptor 4; TLR2: toll-like receptor 2; MAPK: mitogen-activated protein kinase; JAK2: Janus kinase 2; STAT3: signal transducers and activators of transcription 3; PI3K: phosphatidylinositol 3-kinase; Akt: protein kinase B; mTOR: mammalian target of rapamycin; cAMP: cyclic adenosine monophosphate; PKA: protein kinase A; ERK: extracellular signal-regulated kinase; TNF- $\alpha$ : tumor necrosis factor- $\alpha$.

[33-35]; cardiac dysfunction [36]; liver ischemia reperfusioninjury [37]; and endometrial inflammation [38].

It is reported that $\mathrm{AD}$ and $\mathrm{T} 2 \mathrm{DM}$ share many common features including inflammation, oxidative stress, and neuronal degeneration [39]. $\beta$-Amyloid- (A $\beta$-) mediated inflammation plays a critical role in the initiation and progression of $\mathrm{AD}$. HSYA protects $\mathrm{A} \beta$-induced $\mathrm{AD}$ model by inhibiting inflammatory response, which may involve inhibiting the activation of the NF- $\kappa \mathrm{B}$ pathway [32]. The NF- $\kappa$ B signaling pathway will be the first research point for our team in future studies on the antidiabetic mechanisms of HSYA.

Mitogen-activated protein kinase (MAPK) pathway and phosphoinositide 3-kinase/protein kinase $\mathrm{B} /$ mammalian target of rapamycin (PI3K/Akt/mTOR) pathway are two other important signals indicating the intervention of HSYA in the treatment of Parkinson's disease (PD) [40], asthma [41], non-small-cell lung cancer [42], and brain microvascular endothelial injury [43]. It is reported that activated MAPK may be associated with both inflammation and energy metabolism in mice, rats, and humans fed with HFD for a short or long term [44]. A case-control study including 248 cases of T2DM and 101 controls showed that genetic variations in the PI3K/Akt/mTOR signaling pathway may be associated with increasing risk of obesity and diabetes [45]. Our previous discussion showed that HSYA could promote the activation of PI3K/Akt and inhibit the apoptosis of pancreatic $\beta$-cells in HFD- and STZ-induced T2DM rats [13]. Further research on the relationship between HSYA and
PI3K/Akt/mTOR signal in diabetic complications should be conducted.

Other inflammation signals, including toll-like receptor 9 (TLR9) signal and cyclic adenosine monophosphate/protein kinase A (cAMP/PKA) signal, were observed in ischaemic cortex after cerebral ischaemia and reperfusion and acute lung injury $[46,47]$. TLRs are a family of pattern recognition receptors that play a critical role in innate immune response. Recently, studies have reported the important role of TLR4 pathway in insulin resistance [27]. TLRs can be proposed as new targets in the intervention of HSYA in diabetes. The important roles of cAMP/PKA signal in the cognitive impairment of diabetic rats may suggest its involvement in the antidiabetic mechanism of HSYA.

\section{Pharmacokinetic Progressions}

\subsection{Pharmacokinetics of Intravenous Administration}

3.1.1. In Healthy Humans. 36 healthy volunteers were recruited in a single-center, open-label, single-dose, and multiple-dose study. It was found that the area under the curve (AUC) of plasma concentration at different time points and time to peak plasma concentration (Cmax) were linearly related to the dose ranging from 25 to $75 \mathrm{mg}$ in a single administration of HSYA and the elimination half-life was about $3.91-4.18 \mathrm{~h}$. When HSYA was administered for $7 \mathrm{~d}(50 \mathrm{mg} / \mathrm{d})$ continuously, Cmax and AUC decreased significantly and the elimination half-life was prolonged from $3.91 \mathrm{~h}$ to $4.41 \mathrm{~h}$ [48]. 
TABLE 2: The RCT research of SYI (90\% HSYA) in the treatment of diabetes and diabetes complications.

\begin{tabular}{|c|c|c|c|c|c|}
\hline & Diseases & RCT research & Main improved clinical indicators & Mechanism research & Reference \\
\hline \multirow{4}{*}{$\begin{array}{l}\text { Microvascular } \\
\text { complications }\end{array}$} & \multirow{2}{*}{$\begin{array}{l}\text { Diabetic } \\
\text { nephropathy }\end{array}$} & $\begin{array}{l}\text { Early stage: } n=535 \\
\text { (control: } n=532 \text { ) }\end{array}$ & Serum creatinine & $\begin{array}{l}\text { SOD, MDA, TNF- } \alpha \text {, } \\
\text { IL-6, and IL-10 }\end{array}$ & {$[55]$} \\
\hline & & $\begin{array}{l}\text { End stage: } n=50 \\
\text { (control: } n=50 \text { ) }\end{array}$ & $24 \mathrm{~h}$ proteinuria, urea nitrogen & & [56] \\
\hline & $\begin{array}{l}\text { Diabetic } \\
\text { retinopathy }\end{array}$ & $n=92($ control: $n=76)$ & $\begin{array}{l}\text { Serum vascular endothelial growth } \\
\text { factor and endostatin }\end{array}$ & & {$[57]$} \\
\hline & $\begin{array}{c}\text { Diabetic } \\
\text { neuropathy }\end{array}$ & $n=41($ control: $n=41)$ & $\begin{array}{l}\text { Tendon reflexes and EMG nerve } \\
\text { conduction velocity }\end{array}$ & & {$[58]$} \\
\hline \multirow{3}{*}{$\begin{array}{l}\text { Macrovascular } \\
\text { complications }\end{array}$} & $\begin{array}{l}\text { Cardiovascular } \\
\text { disease }\end{array}$ & $\begin{array}{l}\text { Unstable angina pectoris: } \\
n=42 \text { (control: } n=42)\end{array}$ & $\begin{array}{l}\text { Number and duration of angina } \\
\text { pectoris }\end{array}$ & & [59] \\
\hline & $\begin{array}{l}\text { Cerebrovascular } \\
\text { disease }\end{array}$ & $\begin{array}{l}\text { Acute cerebral infarction: } \\
n=40 \text { (control: } n=40)\end{array}$ & NIHSS score & & {$[60]$} \\
\hline & $\begin{array}{c}\text { Peripheral } \\
\text { vascular disease }\end{array}$ & $\begin{array}{l}\text { Diabetic foot ulcers: } n=20 \\
\quad \text { (control: } n=20)\end{array}$ & Wagner classification & & [61] \\
\hline
\end{tabular}

Abbreviations: RCT: randomized controlled trial; SYI: safflower yellow injection; HSYA: hydroxysafflor yellow A; SOD: superoxide dismutase; MDA: malondialdehyde; TNF- $\alpha$ : tumor necrosis factor- $\alpha$; IL-6: interleukin-6; IL-10: interleukin-10; EMG: electromyogram; NIHSS: National Institute of Health Stroke Scale.

Pharmacokinetic studies in healthy humans have shown that the metabolic process in the body after intravenous administration of HSYA conforms to the two-compartment model, indicating that HSYA can be quickly distributed in many organs including the heart, liver, spleen, lungs, brain, intestines, and kidneys [49]. The distribution of HSYA in the kidneys is more than that of the other organs [3]. The excretion of HSYA is mainly from the kidneys, and the cumulative excretion rate of HSYA in urine $24 \mathrm{~h}$ after intravenous administration is up to $88.6 \%[3,49]$. According to the above characteristics, the pharmacokinetic indexes of HSYA in DN patients are different from those of the healthy volunteers.

3.1.2. In Renal Insufficient Patients. It was found that the Cmax and AUC of HSYA in the diabetic impaired renal function group increased and the apparent volume of distribution and clearance rate reduced significantly after a single administration. The results showed that impaired renal function affected pharmacokinetic indicators [50].

Relevant studies have shown that after administering HSYA intravenously for $1 \mathrm{~h}$, the average blood concentration of HSYA in renal insufficient patients was equivalent to 2.64 times that of patients with normal renal functions [51]. It is suggested that the dosage and frequency of administration should be adjusted according to the blood concentration when HSYA is used in DN patients.

3.1.3. In Patients with Traumatic Brain Injury. A sensitive, rapid, and reliable liquid chromatography-tandem mass spectrometry method was applied to investigate the pharmacokinetics of HSYA in patients with traumatic brain injury (TBI). The results demonstrated that some HSYA crossed the blood-brain barrier after administration. This study provides evidence to better understand the pharmacokinetics and potential clinical guidance for TBI treatment [52].

3.1.4. Clinical Antidiabetic Applications. Safflower yellow injection (SYI) containing 90\% HSYA (45 mg HSYA per
$50 \mathrm{mg}$ SYI) has been widely used clinically [53]. In line with clinical guidelines and expert consensus [54], the use of SYI is becoming more and more standardized. Randomized controlled trials (RCTs) of SYI in the treatment of diabetes complications are summarized in Table 2 [55-61].

It can be observed from Table 2 that HSYA has effects on microvascular complications as well as macrovascular complications. Among these complications, HSYA was mostly used in the early stage of DN, and the mechanism research showed that HSYA had anti-inflammatory activity by decreasing TNF- $\alpha$ levels in DN patients [62]. There is no clinical anti-inflammatory research about HSYA on diabetic retinopathy, diabetic neuropathy, etc. The development of further clinical applications of HSYA may need to be carried out alongside its clinical anti-inflammatory effects.

It is said that the age of natural antioxidant compounds in the treatment of diabetic complications is coming [63]. HSYA injection has made some progress in the treatment of different diabetic complications. But from the perspective of patients, it is obvious that an oral drug is more convenient than an injection.

\subsection{Pharmacokinetics of Oral Administration}

3.2.1. In Healthy Humans. The pharmacokinetics of HSYA in 12 healthy volunteers after a single oral administration of HSYA was investigated. The plasma pharmacokinetics of HSYA after oral administration in the 12 healthy subjects showed that the component was absorbed quickly, with a peak time of $1 \mathrm{~h}$ and a short elimination half-life of approximately 2.6-3.5 h [64]. HSYA is relatively polar and easily catabolized and metabolized in the gastrointestinal tract and liver, leading to its rapid elimination, short half-life, and low bioavailability under oral or intragastric administration conditions [49]. The clinical use of HSYA as an oral preparation is being hindered by its low bioavailability, and hence, there is the need for an improvement of its oral bioavailability. 


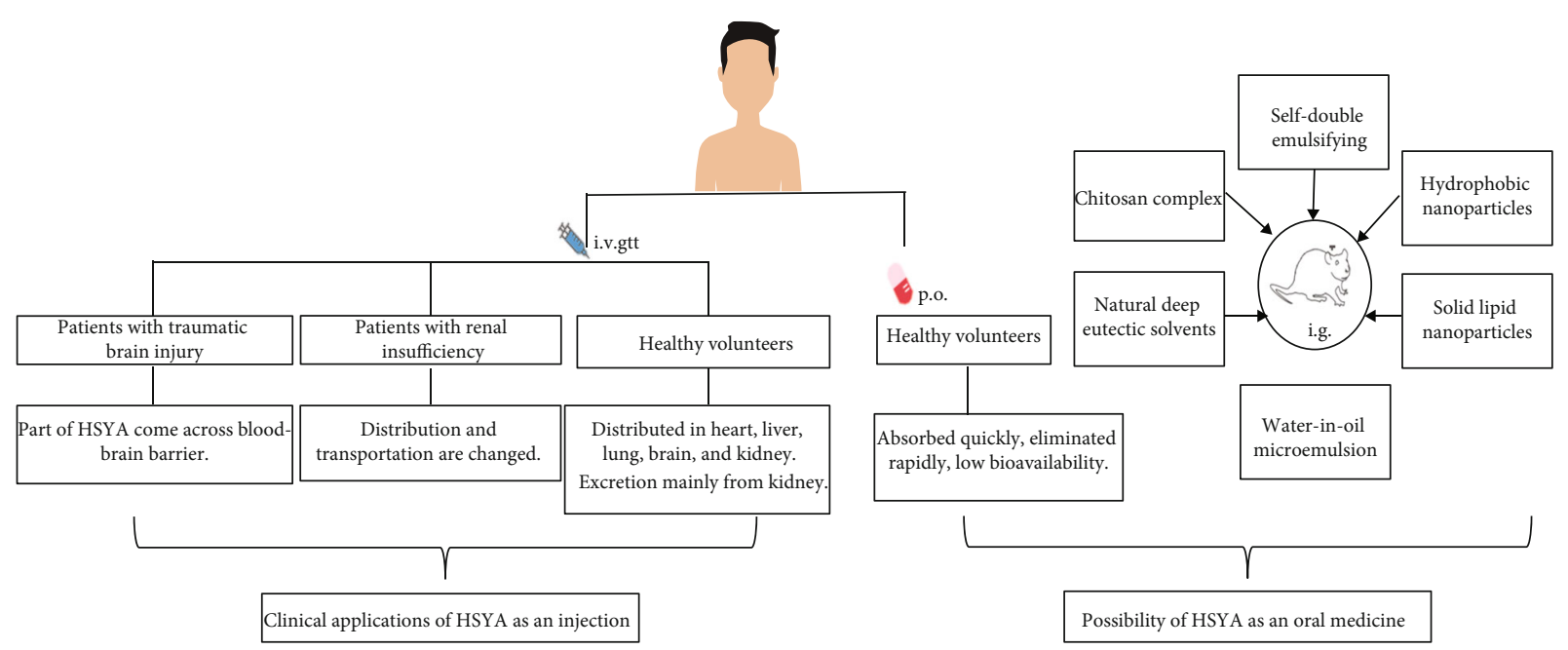

FIGURE 3: Clinical applications and possible medicine development of HSYA based on human and animal pharmacokinetic research. Abbreviation: HSYA: hydroxysafflor yellow A.

3.2.2. Oral Delivery System Research. Fortunately, research on the delivery system of HSYA made it possible to develop it into an oral medicament. Some oral delivery systems of HSYA are shown in Figure 3. They are water-in-oil microemulsion [65], self-double-emulsifying [66], hydrophobic nanoparticles [67], chitosan complex [68], solid lipid nanoparticles [69], and natural deep eutectic solvents [70].

Related research suggested that shell nanoparticles are a highly effective delivery system for resveratrol, another natural compound, due to their significant effects in increasing the bioavailability and anti-inflammation activity [71]. We look forward to a suitable delivery system for HSYA, which will improve not only its bioavailability but also its antiinflammatory activity in the near future.

\section{Conclusion}

HSYA, a major active component from safflower plant, has drawn more interest in recent years for its multiple pharmacological actions. We aim to provide an updated overview of HSYA in diabetes and diabetic complications from these four points: pharmacological actions, molecular mechanisms, pharmacokinetic progressions, and clinical applications. Anti-inflammation mechanism plays an important role in the antidiabetic pharmacological actions of HSYA. Further anti-inflammation research should pay attention to more inflammation signals such as NF- $\kappa \mathrm{B}$ pathway and MAPK pathway. The pharmacokinetic properties of HSYA enhanced its wide clinical use as an injection to treat diabetic complications. Based on the development of drug delivery systems, HSYA could be expected as an oral drug with improved bioavailability and improved anti-inflammatory activity.

\section{Data Availability}

All authors declare that the readers can access the conclusions from the three figures and two tables. All of the figures and tables are summarized based on the references.

\section{Conflicts of Interest}

The authors declare that there is no conflict of interest regarding the publication of this paper.

\section{Authors' Contributions}

Xilan Zhang and Dayue Shen contributed equally to this work.

\section{Acknowledgments}

This study was supported by Wu Jieping Medical Foundation (No. 320.6750.2021-08-10) and Key R \& D Project of Shanxi Province (International Scientific and Technological Cooperation, Independent Topics, No. 201903D421061).

\section{References}

[1] X. Xue, Y. Deng, J. Wang et al., "Hydroxysafflor yellow A, a natural compound from Carthamus tinctorius L with good effect of alleviating atherosclerosis," Phytomedicine, vol. 91, pp. 1-15, 2021.

[2] J. Hou, C. Wang, M. Zhang et al., "Safflower yellow improves the synaptic structural plasticity by ameliorating the disorder of glutamate circulation in $\mathrm{A} \beta_{1-42}$-induced AD model rats," Neurochemical Research, vol. 45, no. 8, pp. 1870-1887, 2020.

[3] F. Zhao, P. Wang, Y. Jiao, X. Zhang, D. Chen, and H. Xu, "Hydroxysafflor yellow A: a systematical review on botanical resources, physicochemical properties, drug delivery system, pharmacokinetics, and pharmacological effects," Frontiers in Pharmacology, vol. 11, pp. 1-21, 2020.

[4] M. Z. Hu, Z. Y. Zhou, Z. Y. Zhou et al., "Effect and safety of hydroxysafflor yellow $A$ for injection in patients with acute ischemic stroke of blood stasis syndrome: a phase II, multicenter, randomized, double-blind, multiple-dose, active-controlled clinical trial," Chinese Journal of Integrative Medicine, vol. 26, no. 6, pp. 420-427, 2020.

[5] Y. H. Wong, S. H. Wong, X. T. Wong et al., "Genetic associated complications of type 2 diabetes mellitus: a review," Panminerva Medical, 2021. 
[6] V. S. Tanwar, M. A. Reddy, and R. Natarajan, "Emerging role of long non-coding RNAs in diabetic vascular complications," Frontiers in Endocrinology (Lausanne), vol. 12, pp. 1-19, 2021.

[7] R. Pradeepa and V. Mohan, "Prevalence of type 2 diabetes and its complications in India and economic costs to the nation," European Journal of Clinical Nutrition, vol. 71, no. 7, pp. 816-824, 2017.

[8] E. D. Canto, A. Ceriello, L. Rydén et al., "Diabetes as a cardiovascular risk factor: an overview of global trends of macro and micro vascular complications," European Journal of Preventive Cardiology, vol. 26, no. 2, pp. 25-32, 2019.

[9] D. Zhou, Z. Huang, X. Zhu, T. Hong, and Y. Zhao, "Combination of endothelial progenitor cells and BB-94 significantly alleviates brain damage in a mouse model of diabetic ischemic stroke," Experimental and Therapeutic Medicine, vol. 22, no. 1, pp. 1-11, 2021.

[10] C. R. L. Cardoso, N. C. Leite, and G. F. Salles, "Importance of hematological parameters for micro- and macrovascular outcomes in patients with type 2 diabetes: the Rio de Janeiro type 2 diabetes cohort study," Cardiovascular Diabetology, vol. 20, no. 1, pp. 1-13, 2021.

[11] R. I. Mota, S. E. Morgan, and E. M. Bahnson, "Diabetic vasculopathy: macro and microvascular injury," Current Pathobiology Reports, vol. 8, no. 1, pp. 1-14, 2020.

[12] M. C. Nwadiugwu, "Inflammatory activities in type 2 diabetes patients with co-morbid angiopathies and exploring beneficial interventions: a systematic review," Frontiers in Public Health, vol. 8, pp. 1-15, 2021.

[13] M. Lee, H. Li, H. Zhao, M. Suo, and D. Liu, "Effects of hydroxysafflor yellow A on the PI3K/AKT pathway and apoptosis of pancreatic $\beta$-cells in type 2 diabetes mellitus rats," Diabetes, Metabolic Syndrome and Obesity: Targets and Therapy, vol. 13, pp. 1097-1107, 2020.

[14] Y. Li, D. Zheng, D. Shen, X. Zhang, X. Zhao, and H. Liao, "Protective effects of two safflower derived compounds, kaempferol and hydroxysafflor yellow A, on hyperglycaemic stressinduced podocyte apoptosis via modulating of macrophage M1/M2 polarization," Journal of Immunology Research, vol. 2020, pp. 1-11, 2020.

[15] H. Liao, Y. Li, X. Zhai et al., "Comparison of inhibitory effects of safflower decoction and safflower injection on protein and mRNA expressions of iNOS and IL- $1 \beta$ in LPS-activated RAW264.7 cells," Journal of Immunology Research, vol. 2019, pp. 1-11, 2019.

[16] M. Lee, H. Zhao, X. Liu et al., "Protective effect of hydroxysafflor yellow A on nephropathy by attenuating oxidative stress and inhibiting apoptosis in induced type 2 diabetes in rat," Oxidative Medicine and Cellular Longevity, vol. 2020, pp. 111,2020

[17] S. Q. Gao, C. Chang, X. Q. Niu, L. J. Li, Y. Zhang, and J. Q. Gao, "Topical application of hydroxysafflor yellow A accelerates the wound healing in streptozotocin induced T1DM rats," European Journal of Pharmacology, vol. 823, pp. 72-78, 2018.

[18] Y. Zhao, H. Sun, X. Li, Y. Zha, and W. Hou, "Hydroxysafflor yellow A attenuates high glucose-induced pancreatic $\beta$-cells oxidative damage via inhibiting JNK/c-jun signaling pathway," Biochemical and Biophysical Research Communications, vol. 505, no. 2, pp. 353-359, 2018.

[19] S. Chen, J. Ma, H. Zhu, S. Deng, M. Gu, and S. Qu, "Hydroxysafflor yellow A attenuates high glucose-induced human umbilical vein endothelial cell dysfunction," Human and Experimental Toxicology, vol. 38, no. 6, pp. 685-693, 2019.
[20] W. Li, J. Liu, P. He et al., "Hydroxysafflor yellow A protects methylglyoxal-induced injury in the cultured human brain microvascular endothelial cells," Neuroscience Letters, vol. 549, pp. 146-150, 2013.

[21] K. Yan, X. Wang, H. Zhu et al., "Safflower yellow improves insulin sensitivity in high-fat diet-induced obese mice by promoting peroxisome proliferator-activated receptor- $\gamma 2$ expression in subcutaneous adipose tissue," Journal of Diabetes Investigation, vol. 11, no. 6, pp. 1457-1469, 2020.

[22] A. Acosta-Montalvo, C. Saponaro, J. Kerr-Conte, J. H. M. Prehn, F. Pattou, and C. Bonner, "Proglucagon-derived peptides expression and secretion in rat insulinoma INS-1 cells," Frontiers in Cell and Developmental Biology, vol. 8, pp. 1-11, 2020.

[23] H. Wang, Y. Zhang, F. Xia, W. Zhang, P. Chen, and G. Yang, "Protective effect of silencing Stat1 on high glucose-induced podocytes injury via Forkhead transcription factor O1regulated the oxidative stress response," BMC Molecular and Cell Biology, vol. 20, no. 1, pp. 1-15, 2019.

[24] H. Chen, Z. Feng, L. Li, and L. Fan, "MicroRNA-9 rescues hyperglycemia-induced endothelial cell dysfunction and promotes arteriogenesis through downregulating Notch1 signaling," Molecular and Cellular Biochemistry, vol. 476, no. 7, pp. 2777-2789, 2021.

[25] J. Hu, L. Zhang, C. Liechty et al., "Long noncoding RNA _GAS5_ regulates macrophage polarization and diabetic wound healing," Journal of Investigative Dermatology, vol. 140, no. 8, pp. 1629-1638, 2020.

[26] J. Zhao, J. Chen, Y. Y. Li, L. L. Xia, and Y. G. Wu, "Bruton's tyrosine kinase regulates macrophage-induced inflammation in the diabetic kidney via NLRP3 inflammasome activation," International Journal of Molecular Medicine, vol. 48, no. 3, pp. 1-12, 2021.

[27] H. Zand, N. Morshedzadeh, and F. Naghashian, "Signaling pathways linking inflammation to insulin resistance," Diabetes and Metabolic Syndrome: Clinical Research and Reviews, vol. 11, Suppl 1, pp. S307-S309, 2017.

[28] R. Sahukari, J. Punabaka, S. Bhasha, V. S. Ganjikunta, S. K. Ramudu, and S. R. Kesireddy, "Plant compounds for the treatment of diabetes, a metabolic disorder: NF- $\kappa$ B as a therapeutic target," Current Pharmaceutical Design, vol. 26, no. 39, pp. 4955-4969, 2020.

[29] X. Yang, L. Chen, Y. Li et al., "Protective effect of hydroxysafflor yellow A on cerebral ischemia reperfusion- injury by regulating GSK $3 \beta$-mediated pathways," Neuroscience Letters, vol. 736, pp. 1-9, 2020.

[30] J. Xu, T. Zhan, W. Zheng et al., "Hydroxysafflor yellow A acutely attenuates blood-brain barrier permeability, oxidative stress, inflammation and apoptosis in traumatic brain injury in rats1," Acta Cirúrgica Brasileira, vol. 35, no. 12, pp. 1-8, 2021.

[31] J. Li, S. Zhang, M. Lu et al., "Hydroxysafflor yellow A suppresses inflammatory responses of BV2 microglia after oxygen-glucose deprivation," Neuroscience Letters, vol. 535, pp. 51-56, 2013.

[32] Z. H. Zhang, L. J. Yu, X. C. Hui et al., "Hydroxy-safflor yellow A attenuates $\mathrm{A} \beta_{1-42}$-induced inflammation by modulating the JAK2/STAT3/NF- $\kappa$ B pathway," Brain Research, vol. 1563, pp. 72-80, 2014.

[33] S. Liu, Y. Wang, H. Wen, X. Sun, and Y. Wang, "Hydroxysafflor yellow A inhibits TNF- $\alpha$-induced inflammation of human fetal lung fibroblasts via NF- $\kappa \mathrm{B}$ signaling pathway," Evidence- 
based Complementary and Alternative Medicine, vol. 2019, pp. 1-9, 2019.

[34] M. Jin, C. J. Xue, Y. Wang et al., "Protective effect of hydroxysafflor yellow A on inflammatory injury in chronic obstructive pulmonary disease rats," Chinese Journal of Integrative Medicine, vol. 25, no. 10, pp. 750-756, 2019.

[35] Y. Zhang, L. Song, R. Pan, J. Gao, B. X. Zang, and M. Jin, "Hydroxysafflor yellow A alleviates lipopolysaccharideinduced acute respiratory distress syndrome in mice," Biological \& Pharmaceutical Bulletin, vol. 40, no. 2, pp. 135-144, 2017.

[36] X. T. Wang, Z. Peng, Y. Y. An et al., "Paeoniflorin and hydroxysafflor yellow A in Xuebijing injection attenuate sepsisinduced cardiac dysfunction and inhibit proinflammatory cytokine production," Frontiers in Pharmacology, vol. 11, pp. 1-19, 2021.

[37] S. Jiang, Z. Shi, C. Li, C. Ma, X. Bai, and C. Wang, "Hydroxysafflor yellow A attenuates ischemia/reperfusion-induced liver injury by suppressing macrophage activation," International Journal of Clinical and Experimental Pathology, vol. 7, no. 5, pp. 2595-2608, 2014.

[38] S. He, X. Wang, Z. Liu et al., "Hydroxysafflor yellow A inhibits staphylococcus aureus-induced mouse endometrial inflammation via TLR2-mediated NF-kB and MAPK pathway," Inflammation, vol. 44, no. 3, pp. 835-845, 2021.

[39] E. Lazar, A. Sherzai, J. Adeghate, and D. Sherzai, "Gut dysbiosis, insulin resistance and Alzheimer's disease: review of a novel approach to neurodegeneration," Frontiers in Bioscience-Scholar, vol. 13, no. 1, pp. 17-29, 2021.

[40] X. Yang, Y. Li, L. Chen et al., "Protective effect of hydroxysafflor yellow A on dopaminergic neurons against 6-hydroxydopamine, activating anti-apoptotic and anti-neuroinflammatory pathways," Pharmaceutical Biology, vol. 58, no. 1, pp. 686-694, 2020.

[41] M. Zheng, X. Guo, R. Pan, J. Gao, B. Zang, and M. Jin, "Hydroxysafflor yellow a alleviates ovalbumin-induced asthma in a guinea pig model by attenuateing the expression of inflammatory cytokines and signal transduction," Frontiers in Pharmacology, vol. 10, pp. 1-12, 2019.

[42] M. Jiang, L. Y. Zhou, N. Xu, and Q. An, "Hydroxysafflor yellow a inhibited lipopolysaccharide-induced non-small cell lung cancer cell proliferation, migration, and invasion by suppressing the PI3K/AKT/mTOR and ERK/MAPK signaling pathways," Thoracic Cancer, vol. 10, no. 6, pp. 1319-1333, 2019.

[43] G. Yang, N. Wang, S. W. Seto, D. Chang, and H. Liang, "Hydroxysafflor yellow a protects brain microvascular endothelial cells against oxygen glucose deprivation/reoxygenation injury: involvement of inhibiting autophagy via class I PI3K/ Akt/mTOR signaling pathway," Brain Research Bulletin, vol. 140, pp. 243-257, 2018.

[44] Z. Wang, M. Zhu, M. Wang et al., "Integrated multiomic analysis reveals the high-fat diet induced activation of the MAPK signaling and inflammation associated metabolic cascades via histone modification in adipose tissues," Frontiers in Genetics, vol. 140, pp. 243-257, 2018.

[45] X. Yin, Z. Xu, Z. Zhang et al., "Association of PI3K/AKT/ mTOR pathway genetic variants with type 2 diabetes mellitus in Chinese," Diabetes Research and Clinical Practice, vol. 128, pp. 127-135, 2017.

[46] Z. Gong, J. Pan, X. Li, H. Wang, L. He, and Y. Peng, "Hydroxysafflor yellow A reprograms TLR9 signalling pathway in ischaemic cortex after cerebral ischaemia and reperfusion,"
CNS \& Neurological Disorders-Drug Targets, vol. 17, no. 5, pp. 370-382, 2018.

[47] C. Wang, Q. Huang, C. Wang et al., "Hydroxysafflor yellow A suppress oleic acid-induced acute lung injury via protein kinase A," Toxicology and Applied Pharmacology, vol. 272, no. 3, pp. 895-904, 2013.

[48] C. Y. Li, J. G. Yin, J. Zhang et al., "Pharmacokinetic profiles of hydroxysafflor yellow A following intravenous administration of its pure preparations in healthy Chinese volunteers," Journal of Ethnopharmacology, vol. 162, pp. 225-230, 2015.

[49] L. Wu, A. Kang, S. J. Le, and Y. P. Tang, "Research progress on process of hydroxyl safflower yellow A in vivo," Chinese Traditional Patent Medicine, vol. 42, no. 1, pp. 150-155, 2020.

[50] G. Lyu, X. Sun, Y. Li, S. Liu, and A. Wang, "Pharmacokinetics of safflower yellow in the diabetes mellitus patients with renal impairment," World Clinical Drugs, vol. 39, no. 9, pp. 625-629, 2018.

[51] J. Wang, F. Liu, C. Xi, M. Li, and H. Gao, "Concentration determination of hydroxysafflor yellow $\mathrm{A}$ in the plasma of the patients with renal insufficiency by HPLC method," Pharmaceutical Care and Research, vol. 16, no. 1, pp. 48-51, 2016.

[52] C. Sheng, W. Peng, Z. Xia, and Y. Wang, "Plasma and cerebrospinal fluid pharmacokinetics of hydroxysafflor yellow A in patients with traumatic brain injury after intravenous administration of Xuebijing using LC-MS/MS method," Xenobiotica, vol. 50, no. 5, pp. 545-551, 2020.

[53] H. Ao, W. Feng, and C. Peng, "Hydroxysafflor yellow A: a promising therapeutic agent for a broad spectrum of diseases," Evidence-based Complementary and Alternative Medicine, vol. 2018, pp. 1-17, 2018.

[54] K. Chen, C. Fu, W. Cong, and Y. Liu, "Chinese expert consensus on the clinical application of safflower yellow," Chinese Journal of Integrated and Traditional and Western Medicine, vol. 37, no. 10, pp. 1167-1173, 2017.

[55] S. Xu, Z. Zhu, Y. Zhang, R. Ye, Q. Feng, and H. Zeng, "Effect of safflower yellow pigment injection on stage III diabetic nephropathy:a systematic review," Traditional Chinese Drug Research \& Clinical Pharmacology, vol. 30, no. 2, pp. 232238, 2019.

[56] S. Wu, B. Zeng, and S. Lin, "Clinical observation of diabetic nephropathy renal failure treated with safflower yellow pigment injection," Chinese Community Doctors, vol. 35, no. 11, pp. 99-101, 2019.

[57] L. Xiong and Y. Dong, "Safflower yellow injection in the treatment of diabetic retinopathy in type 2 diabetes patients," World Clinical Drugs, vol. 35, no. 4, pp. 206-209, 2014.

[58] X. Zhang, "Effect of safflower yellow pigment injection on diabetic peripheral neuropathy," Cardiovascular Disease Journal of Integrated Traditional Chinese and Western Medicine, vol. 4, no. 20, p. 189, 2016.

[59] H. Yang, "Therapeutic effect of safflower yellow pigment injection on type 2 diabetes mellitus complicated with unstable angina pectoris," Chinese Community Doctors, vol. 14, no. 6, p. 210, 2012.

[60] X. Xiong, "Clinical efficacy of safflower yellow injection in the treatment of diabetes with acute cerebral infarction," Journal of Liaoning University of TCM, vol. 20, no. 3, pp. 157-159, 2018.

[61] X. Wu, "40 cases of diabetic foot ulcer treated with safflower yellow pigment injection," Guide of Chinese Medicine, vol. 11, no. 4, pp. 289-290, 2013. 
[62] M. Yin, "Influence of carthamin yellow pigment injection on oxidative stress indexes and inflammatory factors of patients with early diabetic nephropathy," Journal of Clinical Medicine in Practice, vol. 22, no. 23, pp. 51-54, 2018.

[63] T. Caro-Ordieres, G. Marín-Royo, L. Opazo-Ríos et al., “The coming age of flavonoids in the treatment of diabetic complications," Journal of Clinical Medicine, vol. 9, no. 2, pp. 1-30, 2020.

[64] A. D. Wen, J. Yang, Y. Y. Jia et al., "A rapid and sensitive liquid chromatography-tandem mass spectrometry (LC- MS/MS) method for the determination of hydroxysafflor yellow A in human plasma: application to a pharmacokinetic study," Journal of Chromatography B, vol. 876, no. 1, pp. 41-46, 2008.

[65] J. Qi, Q. Zhuang, W. Wu et al., "Enhanced effect and mechanism of water-in-oil microemulsion as an oral delivery system of hydroxysafflor yellow A," International Journal of Nanomedicine, vol. 6, pp. 985-991, 2011.

[66] M. Han, C. Q. Tong, L. Lv, L. Tang, Y. Fang, and J. Q. Gao, "Enhanced absorption of hydroxysafflor yellow A using a self-double-emulsifying drug delivery system: in vitro and in vivo studies," International Journal of Nanomedicine, vol. 7, pp. 4099-4107, 2012.

[67] L. Z. Lv, C. Q. Tong, J. Yu, M. Han, and J. Q. Gao, "Mechanism of enhanced oral absorption of hydrophilic drug incorporated in hydrophobic nanoparticles," International Journal of Nanomedicine, vol. 8, pp. 2709-2717, 2013.

[68] G. N. Ma, F. L. Yu, S. Wang, Z. P. Li, X. Y. Xie, and X. G. Mei, "A novel oral preparation of hydroxysafflor yellow A base on a chitosan complex: a strategy to enhance the oral bioavailability," AAPS PharmSciTech, vol. 16, no. 3, pp. 675-682, 2015.

[69] B. Zhao, S. Gu, Y. Du, M. Shen, X. Liu, and Y. Shen, "Solid lipid nanoparticles as carriers for oral delivery of hydroxysafflor yellow A," International Journal of Pharmaceutics, vol. 535, no. 1-2, pp. 164-171, 2018.

[70] X. Tong, J. Yang, Y. Zhao et al., "Greener extraction process and enhanced in vivo bioavailability of bioactive components from Carthamus tinctorius L. by natural deep eutectic solvents," Food Chemistry, vol. 348, pp. 1-7, 2021.

[71] Y. Liu, X. Liang, Y. Zou, Y. Peng, D. J. McClements, and K. Hu, "Resveratrol-loaded biopolymer core-shell nanoparticles: bioavailability and anti-inflammatory effects," Food \& Function, vol. 11, no. 5, pp. 4014-4025, 2020. 